EPJ manuscript No.

(will be inserted by the editor)

\title{
Effects of immersed moonlets in the ring arc particles of Saturn
}

\author{
G. Madeira ${ }^{1, a}$ and S.M. Giuliatti Winter ${ }^{1}$ \\ Grupo de Dinâmica Orbital \& Planetologia - Univ. of the State of São Paulo - UNESP - \\ Guaratinguetá - Brazil
}

\begin{abstract}
Ring arcs are the result of particles in corotation resonances with nearby satellites. Arcs are present in Saturn and Neptune systems, in Saturn they are also associated with small satellites immersed on them. The satellite Aegaeon is immersed in the G ring arc, and the satellites Anthe and Methone are embedded in arcs named after them. Since most of the population of the arcs is formed by $\mu$ m-sized particles the dissipative effects, such as the plasma drag and the solar radiation force, decrease the lifetime of the arcs. We analysed the effects of the immersed satellites on these arcs by computing the mass production rate and the perturbation caused by them in the arc particles. By comparing the lifetime of the particles and the mass production rate we concluded that Aegaeon, Anthe and Methone did not act as sources for their arcs. We took a step further by analysing a hypothetical scenario formed by an immersed moonlet of different sizes. As a result we found that regardless the size of the hypothetical moonlet (from about 0.10 $\mathrm{km}$ to $4.0 \mathrm{~km}$ ) these moonlets will not act as a source. These arcs are temporary structures and they will disappear in a very short period of time.
\end{abstract}

\section{Introduction}

Corotation resonances between ring particles and nearby satellites are responsible for the formation of arcs in planetary rings. The first arcs to be discovered were the arcs of Neptune Adams ring. These arcs, named Courage, Liberté, Egalité and Fraternité, are the densest parts of the Adams ring [1. Recent data have shown changes in the intensity of these arcs 2] raising the question that these structures can be transient.

The arcs and small satellites of Saturn were discovered by Cassini images: the $\mathrm{G}$ ring arc located between the orbits of the satellites Janus/Epimetheus and Mimas 3 and the arcs of Anthe and Methone, located between the satellites Mimas and Enceladus, immersed in the E ring [4]. These arcs are mainly populated by $\mu \mathrm{m}$-sized dust particles, althoug Cassini data indicate that these structures may have large bodies.

Small satellites are immersed in these arcs. Aegaeon is a satelite of $500 \mathrm{~m}$ in diameter embedded in the $\mathrm{G}$ ring arc [5]. The satellites Anthe [6] and Methone [7]

\footnotetext{
a e-mail: gustavo.o.madeira@unesp.br
} 
are immersed in the arcs named after them with radius of about $500 \mathrm{~m}$ and $1450 \mathrm{~m}$ 8], respectively.

References 5, 6] and 7] have shown that Aegaeon, Methone and Anthe are trapped in a 7:6, 14:15 and 10:11 corotation eccentric resonance (CER) with Mimas, respectively. These arcs are also influenced by nearby Lindblad eccentric resonance (LER). 9] studied the coupling of the corotation and Lindblad resonance through a Hamiltonian formalism, the CoraLin Model, and showed that Aegaeon, Anthe and Methone are close to chaotic regions.

[5] argued that embedded satellites could produce dust due to impacts between interplanetary grains onto the surface of these satellites. These dust particles can replace those particles lost by ejection and collisions. However, 10 showed that despite the material produced by Aegaeon, the $\mathrm{G}$ ring arc is depleted of $\mu$ m-sized particles in less than 40 years due to the dissipative effects of the solar radiation force. Results presented by 11 showed that the arcs of Anthe and Methone lose material produced by their satellites in a few decades also due to dissipative forces.

Through a sample of numerical simulations [11] analysed the system formed by Saturn, Enceladus, Mimas, Anthe and Methone and a set of particles ejected from the surfaces of the small satellites Anthe and Methone. These particles $(<40 \mu \mathrm{m}$ in radius) are also under the effects of the solar radiation force, plasma drag and the electromagnetic force. As a result they found that these particles leave the arcs, in the direction of Enceladus, mainly due to the plasma drag perturbations. The lifetime of these particles is less than few decades.

The Adams ring arcs have also been studied by 12 who proposed a model to confine the arcs. Their model consists of a sample of coorbital satellites azimuthally confining the arc particles, while the satellite Galatea is responsible for radially confining the arc. [13] included in this dynamical system the effects of the solar radiation force and showed that despite the confinement model the particles can become transient and leave the arcs in a very short time.

The goal of this work is to analyse the orbital evolution of a sample of arc particles under the effects of dissipative forces and embedded hypothetical moonlets of different sizes. The moonlet will be responsible for feeding the arcs with dust particles produced by collisions onto its surface, and also for removing the arc particles due to collisions or ejections.

The paper is divided into six sections. The introduction is given in section 1 , while section 2 deals with the behavior of the particles into corotation eccentric resonance. In section 3 the behavior of the arc particles is analyzed under the effects of dissipative forces, such as the solar radiation force and plasma drag. In section 4 we derived three different regimes that the particles can follow depending on the size of the moonlet. Section 5 analysis the fate of the arcs population by comparing the lifetime of the arcs (with and without dissipative forces) with the production of dust by moonlets of different sizes. Our discussion is presented in section 6 .

\section{Conservative dynamics}

The $|m+1|:|m|$ corotation eccentric resonance occurs when the orbit of a particle is closed in the rotating frame with the resonant frequency and the particle is azimuthally confined [14]. Mathematically

$$
(m+1) n_{s a t}-m n-\dot{\varpi}_{s a t}=0
$$

where $n$ and $n_{\text {sat }}$ are the angular frequencies of the particle and the satellite, respectively, and $\dot{\varpi}_{s a t}$ is the derivative of the longitude of the pericentre of the satellite. 
Table 1. Physical parameters of Saturn and the mass of Mimas.

\begin{tabular}{ll}
\hline \hline$J_{2}\left(\times 10^{-6}\right)$ & 16290.544 \\
$J_{4}\left(\times 10^{-6}\right)$ & -936.700 \\
$J_{6}\left(\times 10^{-6}\right)$ & 86.623 \\
$R(\mathrm{~km})$ & 60330 \\
$M_{S}\left(\times 10^{26} \mathrm{~kg}\right)$ & 5.687 \\
$M_{M}\left(\times 10^{19} \mathrm{~kg}\right)$ & 3.754 \\
\hline
\end{tabular}

For external resonances $m$ is taken as a negative integer and positive for internal resonances [14].

In this work we analysed the particles located in the arcs of Aegaeon ( $\mathrm{G}$ ring arc), Methone and Anthe which are in 7:6, 14:15 and 10:11 CER with the satellite Mimas. The resonant angles are [5, 7, 6]:

$$
\begin{gathered}
\phi_{7: 6}=7 \lambda_{M}-6 \lambda-\varpi_{M} \\
\phi_{14: 15}=15 \lambda-14 \lambda_{M}-\varpi_{M} \\
\phi_{10: 11}=11 \lambda-10 \lambda_{M}-\varpi_{M}
\end{gathered}
$$

where $\lambda$ is the mean longitude and $\varpi$ is the longitude of the pericentre. The subscript $M$ refers to Mimas and no subscript is related to the arc particle.

First of all we numerically simulated a sample of particles under the gravitational effects of Saturn and its gravity coefficients $\left(J_{2}, J_{4}\right.$ and $\left.J_{6}\right)$, and the satellite Mimas. The adopted parameters of Mimas are semi-major axis $a_{M}=185539 \mathrm{~km}$, eccentricity $e_{M}=0.0196$ and inclination $I_{M}=1.574^{\circ}$, obtained from JPL-Horizons System (2451545.5 JD); the angular parameters were assumed equal to zero. Table 1 presents Saturn physical parameters: radius $\left(R_{S}\right.$ in $\left.\mathrm{km}\right)$ and mass $\left(M_{M}\right.$ in $\left.\mathrm{kg}\right) \underline{8}$, and the gravity coefficients $\left(J_{2}, J_{4}\right.$ and $\left.J_{6}\right)$ derived from [5], and the mass of Mimas in $\mathrm{kg}$ (from JPL-Horizons System).

The angular frequency and semimajor axis are related by the keplerian third law $\left(G M_{S}=n^{2} a^{3}\right.$, where $\left.G=6.674184 \times 10^{-11} \mathrm{~m}^{3} \mathrm{~kg}^{-1} \mathrm{~s}^{-2}\right)$ and the semimajor axis resonant $\left(a_{c}\right)$ can be determinated numerically by the Newton-Raphson iterative method [15]:

$$
a_{n+1}=a_{n}-\frac{\dot{\phi}}{\frac{d \dot{\phi}\left(a_{n}\right)}{d a}}
$$

where $a_{n}$ and $a_{n+1}$ are the semimajor axis found after the $n_{\text {th }}$ and $n_{+1-\text { th }}$ iteration, respectively. We assumed an error of $10^{-3} \mathrm{~km}$ and the initial condition of iteration as:

$$
a_{0}=\left(\frac{m}{m+1}\right)^{\frac{2}{3}} a_{M}
$$

The width of the resonance $\left(W_{c}\right)$ is given by [16]:

$$
W_{c}=8 a_{c} \sqrt{\frac{a_{M}\left|R^{(c)}\right|}{3 G M_{S}}}
$$
[17]:

The term $R^{(c)}$ is the term of the disturbing function associated with the CER

$$
R^{(c)}=\frac{G M_{M} e_{M}}{2 a_{M}} \cos \phi\left[2 m+1+a_{c} \frac{d}{d a_{c}}\right] b_{1 / 2}^{(m)}
$$


Table 2. Central geometric semimajor axis $\left(a_{c}\right)$ of the resonances and the resonance width $\left(W_{c}\right)$.

\begin{tabular}{lcc}
\hline & $a_{c}(\mathrm{~km})$ & $W_{c}(\mathrm{~km})$ \\
\hline $7: 6$ & 167493.5 & 64.0 \\
$14: 15$ & 194232.9 & 111.4 \\
$10: 11$ & 197655.5 & 96.3 \\
\hline
\end{tabular}

where $b_{1 / 2}^{(m)}$ is the Laplace coefficient.

The semi-major axis resonant (Equation 5) and the width of the resonance (Equation 7) are presented in Table 2

The particles were assumed to be in almost circular orbits with $e=10^{-5}$ orbiting in the equatorial plane of Saturn. A sample of particles were uniformly distributed in the range $\left[a_{c}-W_{c} / 2, a_{c}+W_{c} / 2\right]$ and mean longitude $\left[0^{\circ}, 360^{\circ}\right]$, each $1 \mathrm{~km}$ and $1^{\circ}$. The time span of the numerical simulation is 100 years. We used the Mercury integrator [18] with the Burlish-Stöer algorithm. The algorithm described in [19] was used to convert the state vector into the geometric elements in order to remove the short variations caused by the gravity coefficients.

The 7:6, 14:15 and 10:11 CER produce 6, 15 and 11 equilibrium points, respectively, where the particles move around in the rotating frame with the resonant frequency. The behaviour of the resonant angle of the set of particles has been verified. Figure 1 shows the initial semimajor axis and mean longitude of the particles whose resonant angle librates (as shown in black) and in gray are those particles which resonant angle is circulating.

Due to the nearby Lindblad resonance, the particles orbits are excited [9] to values of the same order of those observed in the arcs as can be seen in Figure 2, 4, 5]. This figure shows the time variation of the eccentricity of a representative particle in $7: 6$, 14:15 and 10:11 CER. We verified that the particles remain azimuthally trapped for a time longer than $10^{5}$ years.

\section{Dissipative Forces}

The ring arcs are most composed by $\mu \mathrm{m}$-sized particles of $1 \mu \mathrm{m}$ to $10 \mu \mathrm{m}$ in radius $(r)$, as observed by Cassini spacecraft counters 4, 5. Solar radiation force and plasma drag are dissipative forces that can alter the motion of these tiny particles. Therefore we add these forces to the dynamical system in order to analyse the lifetime of the arcs.

The force experienced by a circumplanetary particle due to the solar radiation force (SRF) is given by 20 ]

$$
\mathbf{F}_{S R F}=\frac{\Phi A}{c} Q_{p r}\left\{\left[1-\frac{\mathbf{r}_{s p}}{r_{s p}} \cdot\left(\frac{\mathbf{V}_{P}}{c}+\frac{\mathbf{V}}{c}\right)\right] \frac{\mathbf{r}_{s p}}{r_{s p}}-\frac{\mathbf{V}_{P}+\mathbf{V}}{c}\right\}
$$

where $c$ is the speed of light, $A$ is the cross section of the particles, which are considered to be made of an ideal material $\left(Q_{P R}=1\right)$ and $\mathbf{V}$ is the velocity vector of the particle with respect to the planet. The planet is considered to be in a circular heliocentric orbit with position $r_{s p}\left(r_{s p}=\left|\mathbf{r}_{s p}\right|\right)$ and velocity $\mathbf{V}_{P}$, and $\Phi$ is the solar flux. Secondary effects such as the Yarkovsky effect, planetary light deflection and shadow were neglected [21].

The solar radiation force is composed by two components: the Poynting-Robertson drag and the radiation pressure. The Poynting-Robertson component (the terms that 


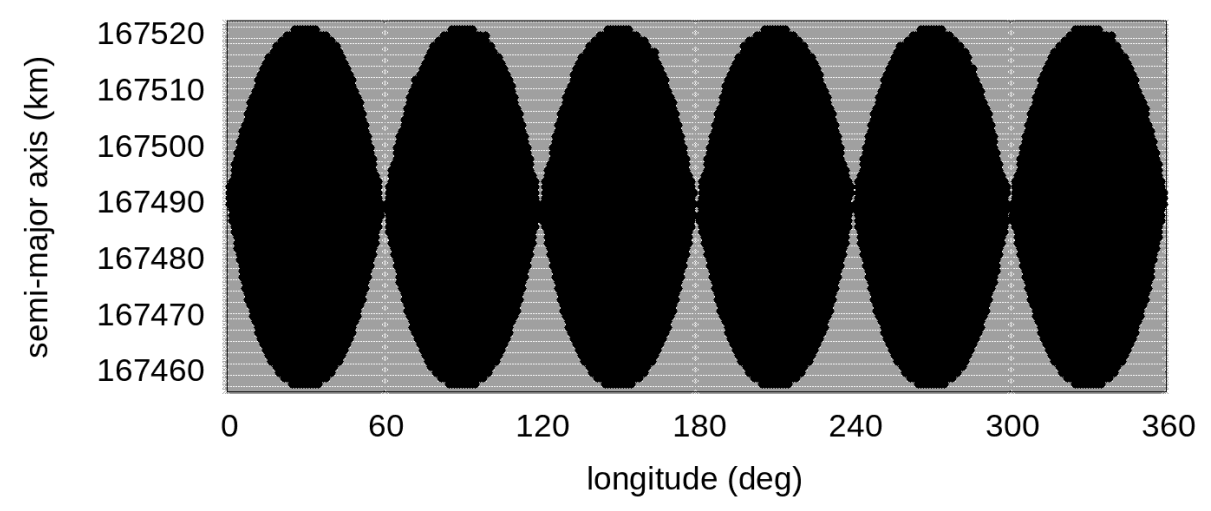

(a)

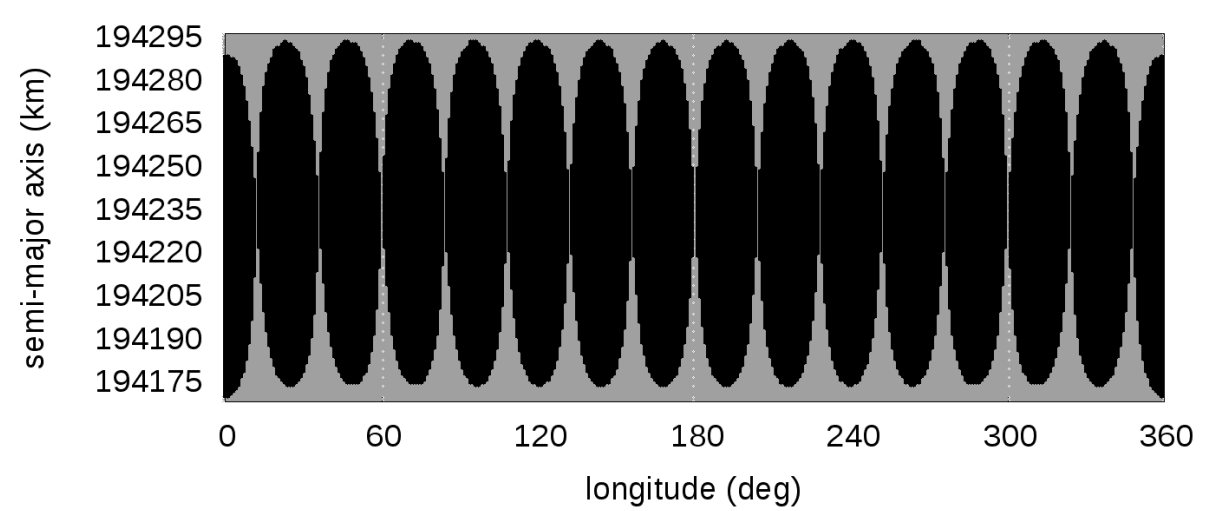

(b)

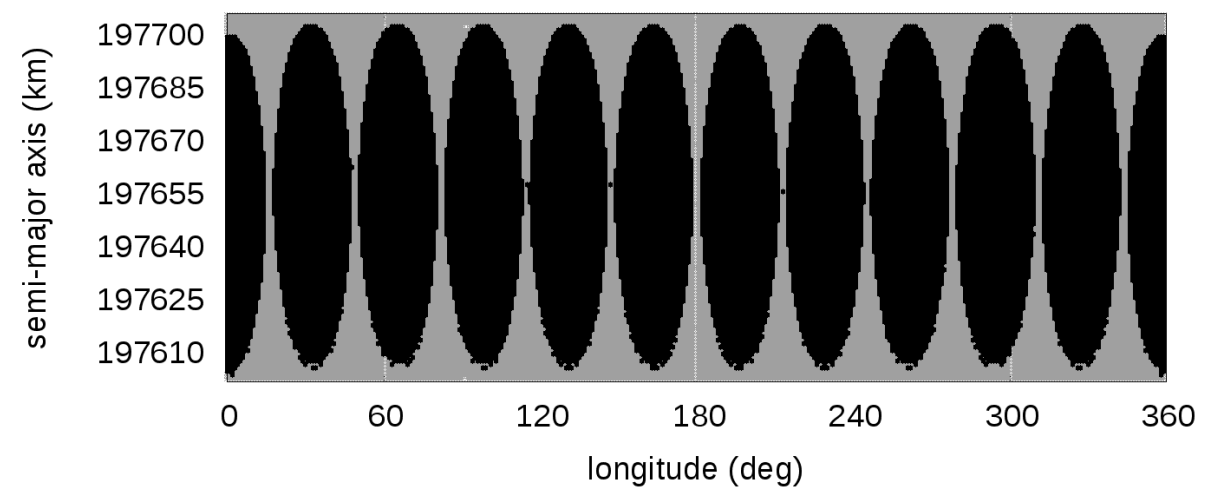

(c)

Fig. 1. In black are shown those particles in resonance with the satellite Mimas: a) 7:6, b) 14:15 and c) 10:11 CER. 


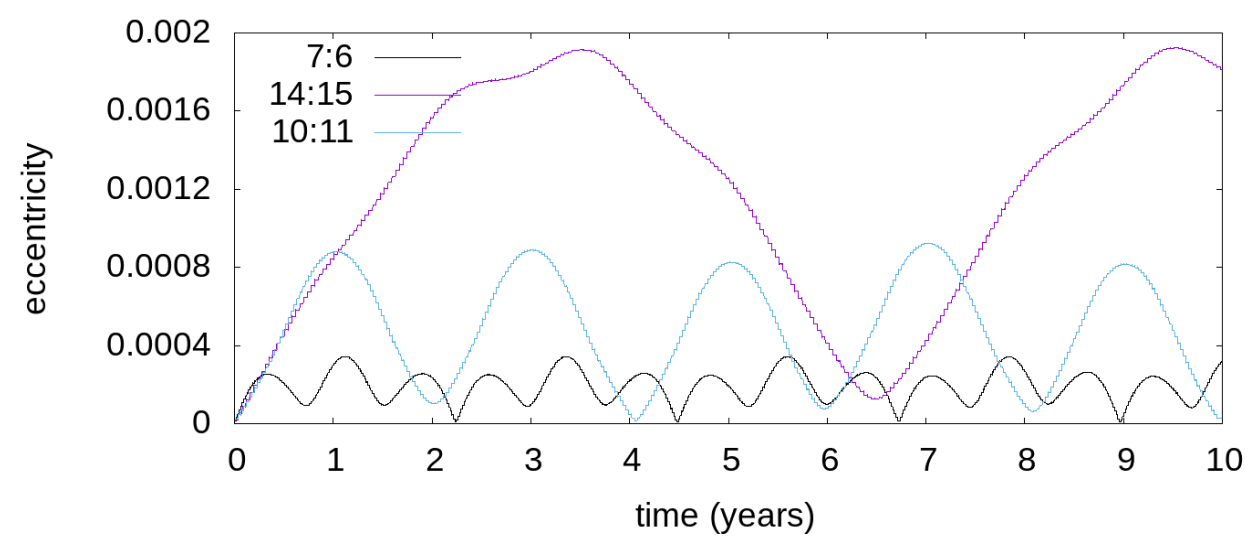

Fig. 2. Time variation of the eccentricity of a representative particle in 7:6, 14:15 and 10:11 CER under the influency of a nearby Lindblad resonance.

depend on the velocities in equation 9 provokes a decreasing in the semi-major axis of the particles and the radiation pressure component causes an excitation of the orbits [22. The radiation pressure is also responsible for small period variations in the semi-major axis of the particles. These oscillations are responsible for removing them from the CER [10.

Another dissipative force is the plasma drag. Data obtained by Cassini mission showed that the satellite Enceladus ejects water vapour through geysers near to its south pole. These geysers correspond to the largest source of water group ions $\left(\mathrm{O}^{+}\right.$, $\mathrm{OH}^{+}, \mathrm{H}_{2} \mathrm{O}^{+}$and $\left.\mathrm{H}_{3} \mathrm{O}^{+}\right)$for the saturnian plasma bulk. The $\mathrm{O}^{+}$ion is the most abundant one 23 .

Therefore, $\mu \mathrm{m}$-sized particles located in the arcs of Methone and Anthe collide with the plasma material, experiencing the plasma drag (PD) [24]

$$
\mathbf{F}_{P D}=\pi n_{i} m_{i} u_{i}^{2} r^{2}\left[\left(M_{i}+\frac{1}{2 M_{i}}\right) \frac{\exp -M_{i}^{2}}{\sqrt{\pi}}+\left(M_{i}^{2}+1-\frac{1}{4 M_{i}^{2}}\right) \operatorname{erf} M_{i}\right] \hat{u}_{t}
$$

where $n_{i}, u_{i}$ and $m_{i}$ are the number density, thermal velocity and mass, respectively, of the ions and $M_{i}$ is the Mach number. We assumed the value of $M_{i}$ relative to $\mathrm{O}^{+}$ to be 1.2 and $n_{i}=43.2 \mathrm{~cm}^{3}$ in the $14: 15$ region and $n_{i}=44 \mathrm{~cm}^{3}$ in the 10:11 region [1]. $\hat{u}_{T}$ is the unity vector in the tangential direction of the orbit of the particle.

The indirect term generated by the interaction of the plasma material due to Coulomb forces was disregarded, once it is, at least, one order of magnitude weaker [1].

The plasma drag causes an increasing in the semi-major axis of the particles and also changes in the eccentricities 24]. In the arcs of Anthe and Methone this increasing in the semi-major axis is about $\frac{600}{r(\mu \mathrm{m})} \mathrm{km} /$ year [1].

We numerically simulated one site of each resonance considering particles with sizes of $1 \mu \mathrm{m}$ and $10 \mu \mathrm{m}$ for 1000 years. The particles are under the gravitational effects of Saturn (with the gravity coefficients) and Mimas, and the solar radiation force. Since the effects of the plasma drag on the particles located in the 7:6 CER are two orders of magnitude weaker, we included this dissipative force only in the arcs of Anthe and Methone.

Figure 3 shows a) the difference between the semi-major axis of the particle and the centre of the site $(\Delta a)$ and b) the resonant argument $(\phi)$ as a function of time. The $1 \mu \mathrm{m}$ sized particle is initially located at $30 \mathrm{~km}$ and $2^{\circ}$ from the centre of the 7:6 


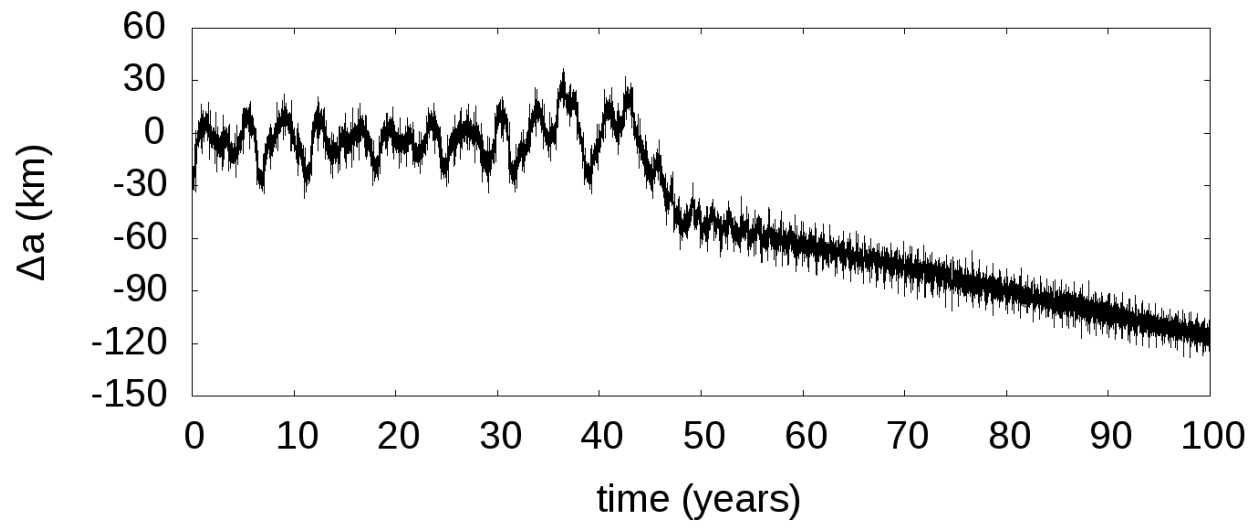

(a)

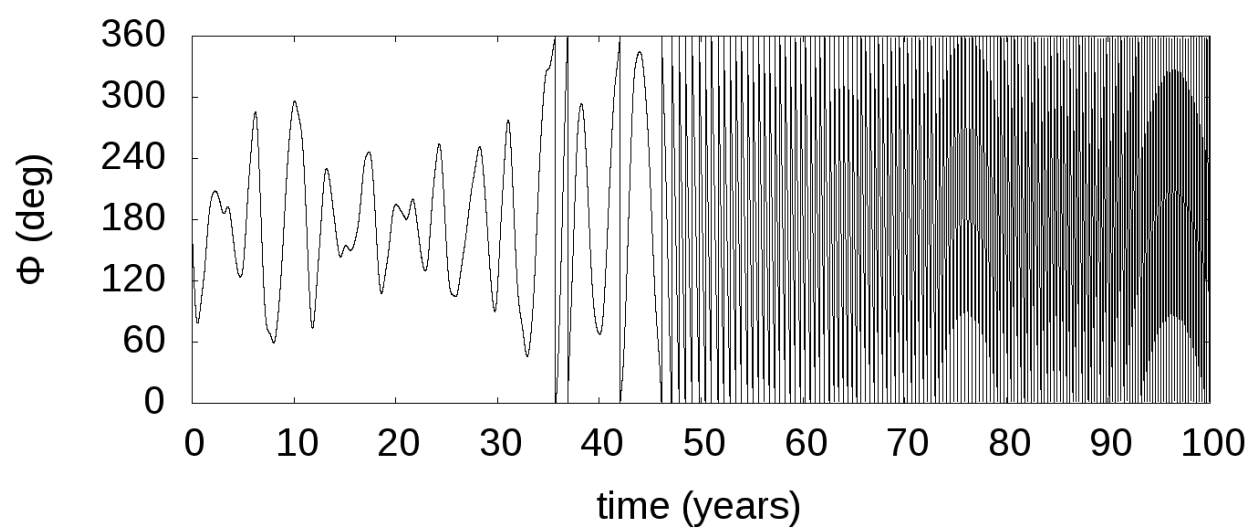

(b)

Fig. 3. Time variation of a) $\Delta a$ and b) $\phi$, where $a_{c}=167493.5 \mathrm{~km}$ for a $1 \mu \mathrm{m}$ sized particle initially at $\Delta a=-30 \mathrm{~km}$ and $\Delta \lambda=2^{\circ}$ from the centre of 7:6 CER site.

CER site. The particle leaves the resonance after 35 years due to the solar radiation force. It is trapped again in resonance and leaves the 7:6 resonance in about 45 years. As can be seen in Figure 3 a the semimajor axis start decreasing due to the PoyntingRobertson component. Similar results were discussed in [10].

Figure 4 presents the variation of $\Delta a$ and $\phi$ for a $10 \mu \mathrm{m}$ sized particle located at the 10:11 CER site. The particle is initially at $\Delta a=-10 \mathrm{~km}$ and $\Delta \lambda=0^{\circ}$ from the centre of the resonance. In Figures $4 a$ and $4 \mathrm{~b}$ there are two curves: in the top the particle is disturbed by the solar radiation force and the plasma drag while in the bottom the particle is only under the effects of the solar radiation force. The particle leaves the resonance after 10 years. As can be seen in Figure 4 , when both effects, solar radiation force and plasma drag, are present in the system, the particle leaves the resonance faster if only the solar radiation force is included. The semi-major axis starts increasing after the particles leaves the resonance due to the plasma drag. This representative particle goes to the outer edge of the $\mathrm{E}$ ring.

Table 3 gives the lifetime of the CER sites due to the dissipative forces. The lifetime is defined as the time to $90 \%$ of the initial sample of particles leave the CER or collide with the embedded moonlets, when moonlets are located in the system 


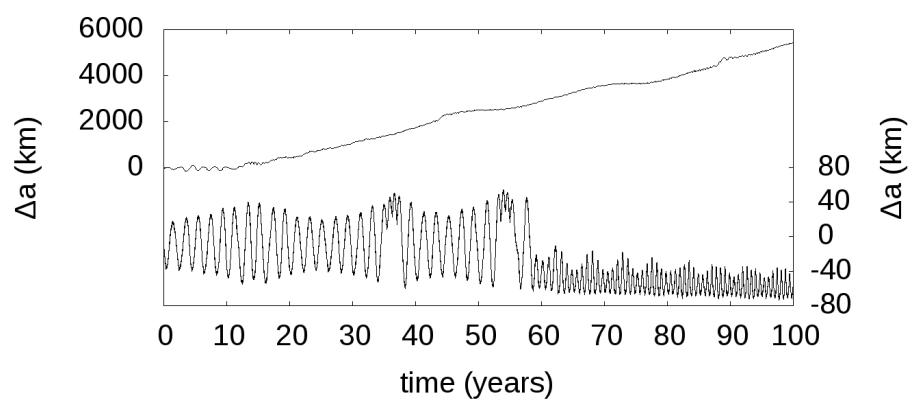

(a)

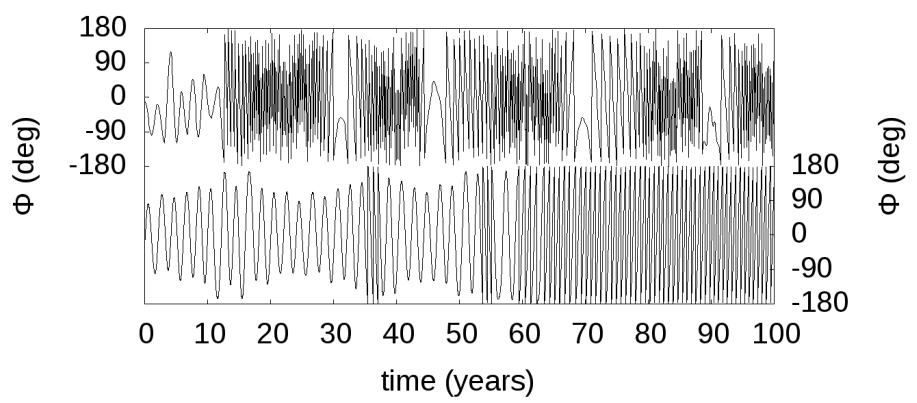

(b)

Fig. 4. Time variation of a) $\Delta a$ and b) $\phi$ for a $10 \mu \mathrm{m}$ sized particles initially located at $\Delta a=-10 \mathrm{~km}$ and $\Delta \lambda=0$ from the centre of the 10:11 CER site. In each figure (a and b) there are two curves: in the top the particle is disturbed by the solar radiation force and the plasma drag while in the bottom the particle is only under the effects of the solar radiation force.

Table 3. Time (in years) to $90 \%$ of the particles leave each arc.

\begin{tabular}{cccccc}
\hline \multicolumn{2}{c}{$7: 6$} & \multicolumn{2}{c}{$14: 15$} & \multicolumn{2}{c}{$10: 11$} \\
$1 \mu \mathrm{m}$ & $10 \mu \mathrm{m}$ & $1 \mu \mathrm{m}$ & $10 \mu \mathrm{m}$ & $1 \mu \mathrm{m}$ & $10 \mu \mathrm{m}$ \\
\hline 150 & $>1000$ & 10 & 80 & 5 & 34 \\
\hline
\end{tabular}

(section 4). Since the particles located at the 7:6 CER are only under the effects of the solar radiation their lifetimes are larger compared to the lifetimes of the particles at the 14:15 and 10:11 CER. Since the particles at 10:11 CER are closer to Enceladus (semi-major axis $\sim 238000 \mathrm{~km}[11$ ) they have the smallest lifetime of the three systems due to the effects of the plasma drag. The $1 \mu \mathrm{m}$ sized particles at the 14:15 and 10:11 CER last less than a decade.

Although the large majority of the particles are those represented in Figures 3 and 4 a few of them may transit between the resonant arcs as shown in Figures 5 and 6. These figures present the time variation of the azimuthal angle $(\theta)$ in the rotating reference frame with the same velocity of the resonant frequency and the resonance angle $(\phi)$ of a $10 \mu \mathrm{m}$ sized particle that leaves and returns to the resonance. In Figure 5 the particle is initially at $\Delta a=-40 \mathrm{~km}$ and $\Delta \lambda=17^{\circ}$ from the 10:11 CER site and in Figure 6 the particle is initially at $\Delta a=70 \mathrm{~km}$ and $\Delta \lambda=0^{\circ}$ from the 14:15 CER site. As can be seen through the time variation of $\theta$ and $\phi$, when the resonant angle 


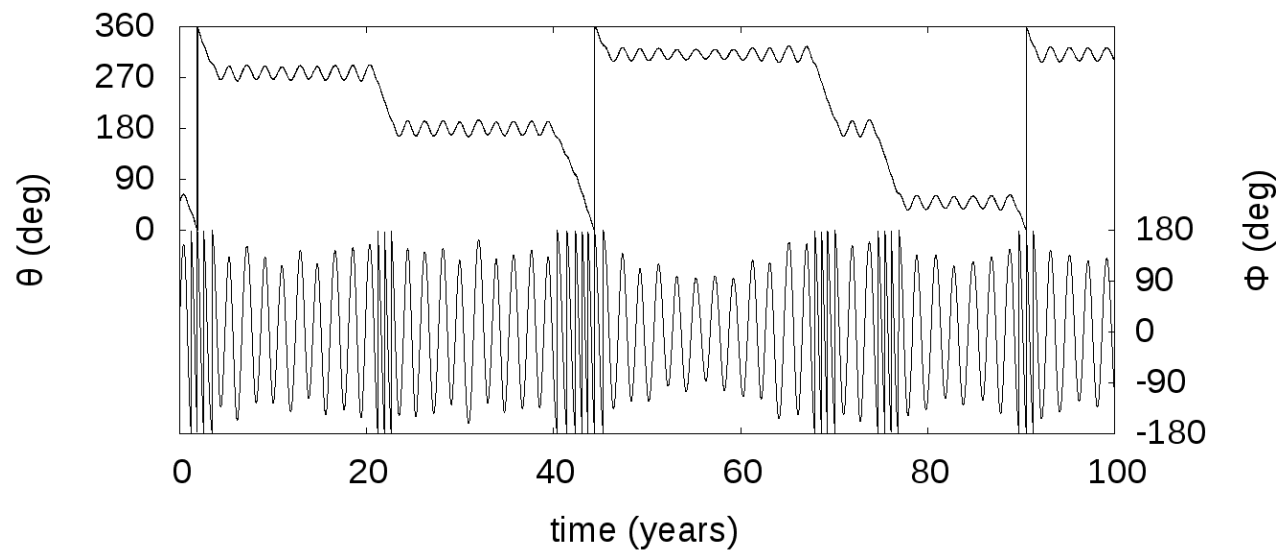

Fig. 5. Time variation of $\theta$ and $\phi$. The $10 \mu \mathrm{m}$ sized particle is initially at $\Delta a=-40 \mathrm{~km}$ and $\Delta \lambda=17^{\circ}$ from the centre of a 10:11 CER site.

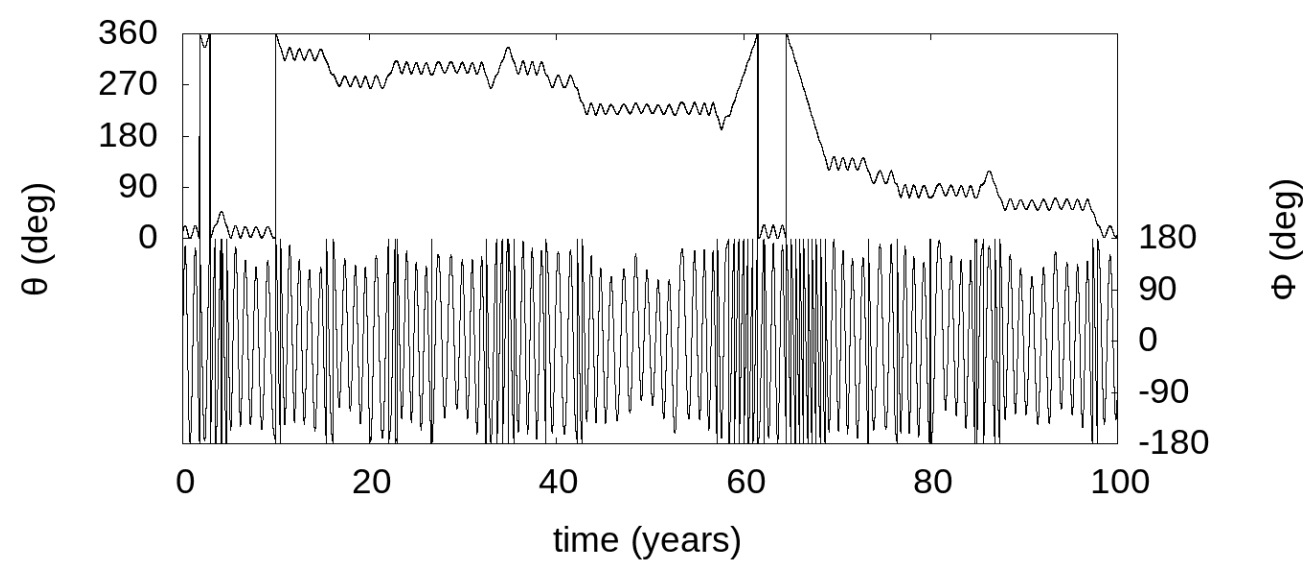

Fig. 6. Time variation of $\theta$ and $\phi$. The $10 \mu \mathrm{m}$ sized particle is initially at $\Delta a=70 \mathrm{~km}$ and $\Delta \lambda=0^{\circ}$ from the centre of a 14:15 CER site.

$\phi$ librates the particles can be located in different values of $\theta$. These are examples of particles travelling between the arcs.

\section{Immersed Moonlets}

In this section are analysed the effects caused by an icy moonlet of different sizes located at the equilibrium point in the centre of the 7:6 CER site. The ring arc particles are under the effects of Saturn and its gravity coefficients $\left(J_{2}, J_{4}\right.$ and $\left.J_{6}\right)$, the satellite Mimas and a hypothetical embedded moonlet. Its mass $m$ was assumed to be a fraction of Mimas' mass $\left(\mu=m / M_{M}\right)$, varying from $10^{-10}(R \sim 100 \mathrm{~m})$ to $10^{0}(R \sim 200 \mathrm{~km})$. The density of the moonlet is assumed to be $1 \mathrm{~g} / \mathrm{cm}^{3}$.

We numerically simulated the system using the Mercury integrator [18, with the Burlish Stöer algorithm. The ring arc is formed by a sample of 200 particles randomly chosen from the arc analysed in the last section.

Figure 7 shows the azimuthal angle $\theta$ of a particle initially located at $\Delta \lambda=2^{\circ}$ from the centre of the 7:6 CER site. The moonlet is at the centre of the site. Different values 


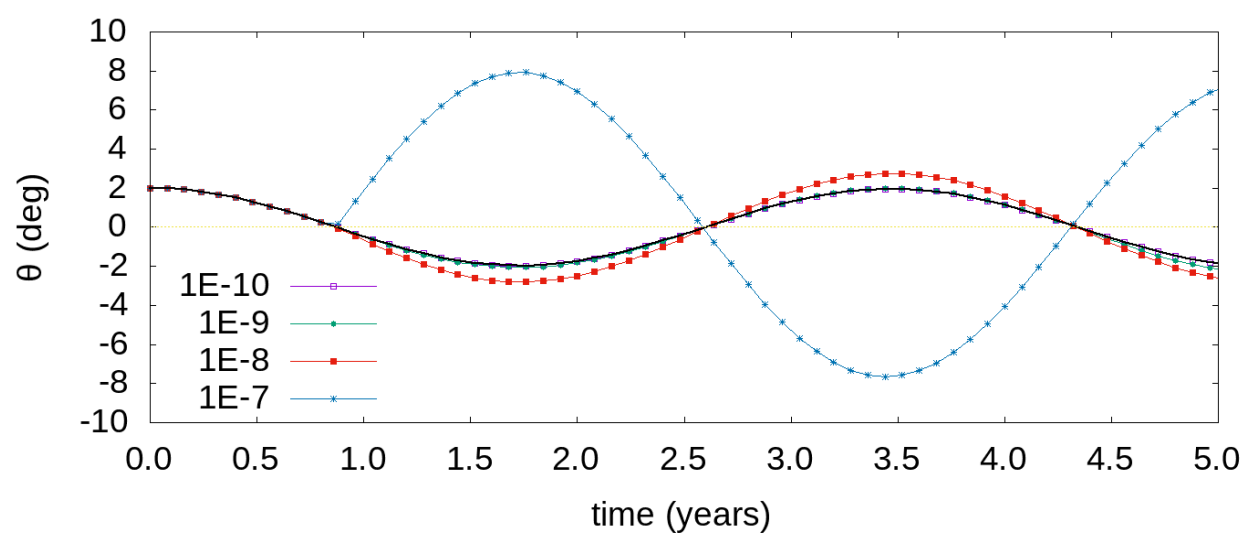

(a)

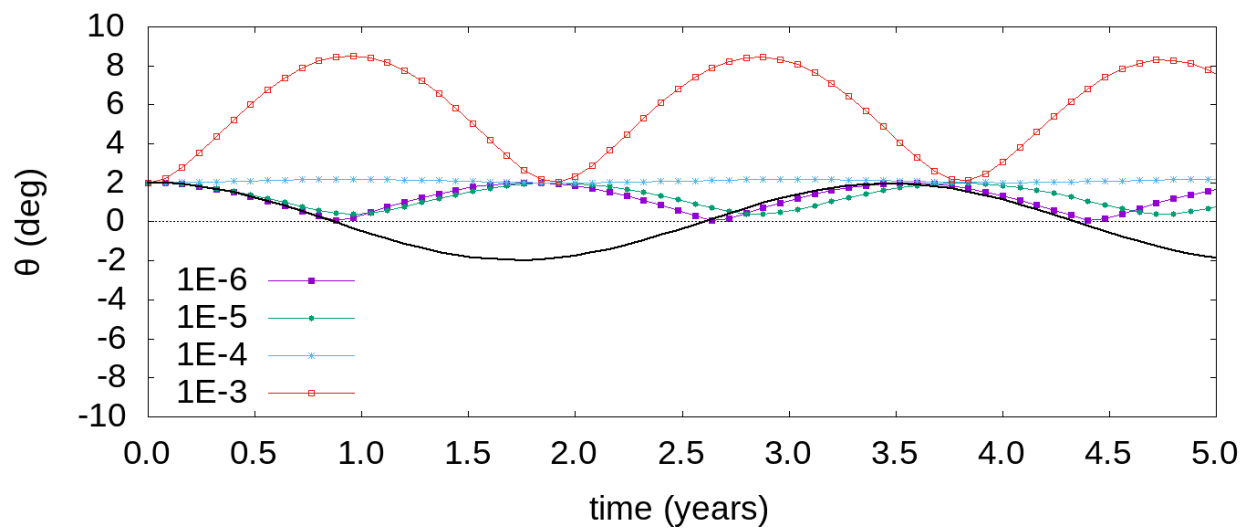

(b)

Fig. 7. Variation of the azimuthal angle $\theta$ of a particle initially displaced $2^{\circ}$ from the centre of the corotation site for different values of $\mu$. The full line represents this variation when no immersed moonlet is located in the site. The presence of the moonlet can generate different regimes: a) first regime: for the mass ratios of $10^{-10}$ and $10^{-9}$ the two curves overlapp, and b) second regime.

of $\mu$ give rise to three distinct regimes. The black thicker line shows the variation of $\theta$ when the moonlet is not in the system. Moonlets with $\mu=10^{-10}-10^{-7}$, which we called the first regime, provoke an increasing in the excursions of the ring arc particles around the CER equilibrium point. In Figure 7 , which corresponds to the first regime, the azimuthal angle of the particle increases after a close approach with the moonlet in less than one year. Larger moonlets induce larger variations in $\theta$.

Moonlets ranging from $10^{-6}$ to $10^{-3}$, second regime, are responsible for azimuthally confining the particles. These particles stay confined in half part of the arc. In Figure $7 \mathrm{~b}$, second regime, the perturbation of the moonlet provokes a change in the motion of the particles. The particles did not move around the CER equilibrium point but remain located between the moonlet and the edge of the arc. In the third regime which corresponds to a moonlet with $\mu \geq 10^{-3}$ the perturbation of the moonlet removes the particle from the resonance with Mimas. The particle stays in a horseshoe orbit with the moonlet (see figure 8f). 
The motion of the particles in the rotating reference system can be seen in Figure 8 . Three values of $\mu$ are shown: a) $\mu=10^{-8}$ corresponding to a moonlet with radius of $500 \mathrm{~m}, \mathrm{~b}) \mu=10^{-5}$ corresponding to a moonlet with radius of $5 \mathrm{~km}$ and c) $\mu=10^{-2}$ corresponding to a moonlet with radius of $50 \mathrm{~km}$. The dot shows the position of the hypothetical moonlet. The motion of the particle in the system formed only by Saturn and Mimas is shown in full line (the smallest arc), when only Saturn and the moonlet are in the system the behaviour of the particle is represented by a dashed line (a horseshoe orbit) and when both perturbers are present, Mimas and the moonlet, the motion of the particle can be seen in a thicker full line.

In Figure 8a the particle starts its motion under the gravitational effects of Mimas. However due to the moonlet the particle completes an arc larger than if it was only under the effects of Mimas. In Figure $8 \mathrm{~b}$ as the particle approaches the moonlet it returns to a small arc becoming confined in half part of the corotation site. In Figure 8 the particle is removed from the site due to the effects of the large moonlet and becomes confined in a horseshoe orbit. The perturbation of Mimas causes only small variations in the horseshoe orbit.

Similar results were found for the 14:15 and 10:11 CER sites when an immersed moonlet is located in its centre.

\section{Fate of the CER Sites}

Immersed moonlets on planetary arcs or rings can help to replenish those particles lost by collisions or ejection from the system. Interplanetary dust particles (IDPs) can collide with these moonlets; these collisions produce material which can be ejected from their surfaces. These IDPs, originate from the Jupiter family and the Kuiper Belt, travel through the interplanetary environment with velocities of order of kilometres per second 25.

Following the algorithm presented by [26], the mass production rate $\left(M^{+}\right)$given by a moonlet with radius $R$ is

$$
M^{+}=\pi R^{2} F_{i m p} Y
$$

where $F_{\mathrm{imp}}$ is the mass flux of impactors and $Y$ is the ejecta yield.

Reference 25] estimates that the impactors mass flux at Saturn's region is of order $F_{i m p}^{\infty}=10^{-17} \mathrm{~kg} /\left(\mathrm{m}^{2} \mathrm{~s}\right)$. The mean velocity of the impactors is $v_{i m p}^{\infty}=9.5 \mathrm{~km} / \mathrm{s}$ 27. As the impactors approach the planet, the mass flux and also the velocity are enhanced due to the gravitational focusing of the planet. At distance $a$ from Saturn's centre, the impactor mass flux $F_{i m p}$ and $v_{i m p}$ are [28]

$$
\begin{gathered}
\frac{v_{i m p}}{v_{i m p}^{\infty}}=\sqrt{1+\frac{2 G M_{S}}{a\left(v_{i m p}^{\infty}\right)^{2}}} \\
\frac{F_{i m p}}{F_{i m p}^{\infty}}=\frac{1}{2}\left(\frac{v_{i m p}}{v_{i m p}^{\infty}}\right)^{2}+\frac{1}{2} \frac{v_{i m p}}{v_{i m p}^{\infty}}\left[\left(\frac{v_{i m p}}{v_{i m p}^{\infty}}\right)^{2}-\left(\frac{R_{S}}{a}\right)^{2}\left(1+\frac{2 G M_{S}}{R_{S}\left(v_{i m p}^{\infty}\right)^{2}}\right)\right]^{1 / 2}
\end{gathered}
$$

The ejecta yield depends on the composition of the moonlet. 29] found that the ejecta yield for icy satellites hit by $\mu \mathrm{m}$-sized projectiles is

$$
Y=2.64 \times 10^{-5} m_{i m p}^{0.23} v_{i m p}^{2.46}
$$

where $m_{i m p}$ is the mass of the impactor. This value was assumed to be $10^{-18} \mathrm{~kg}$ in Saturn's region [27]. 


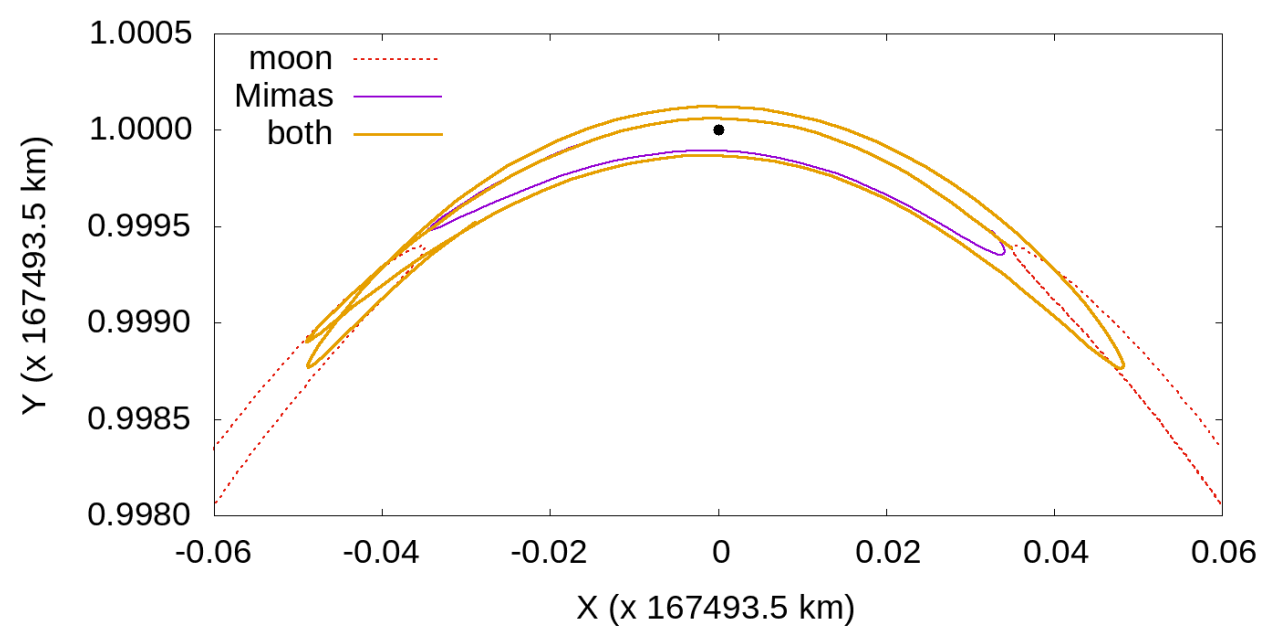

(a)

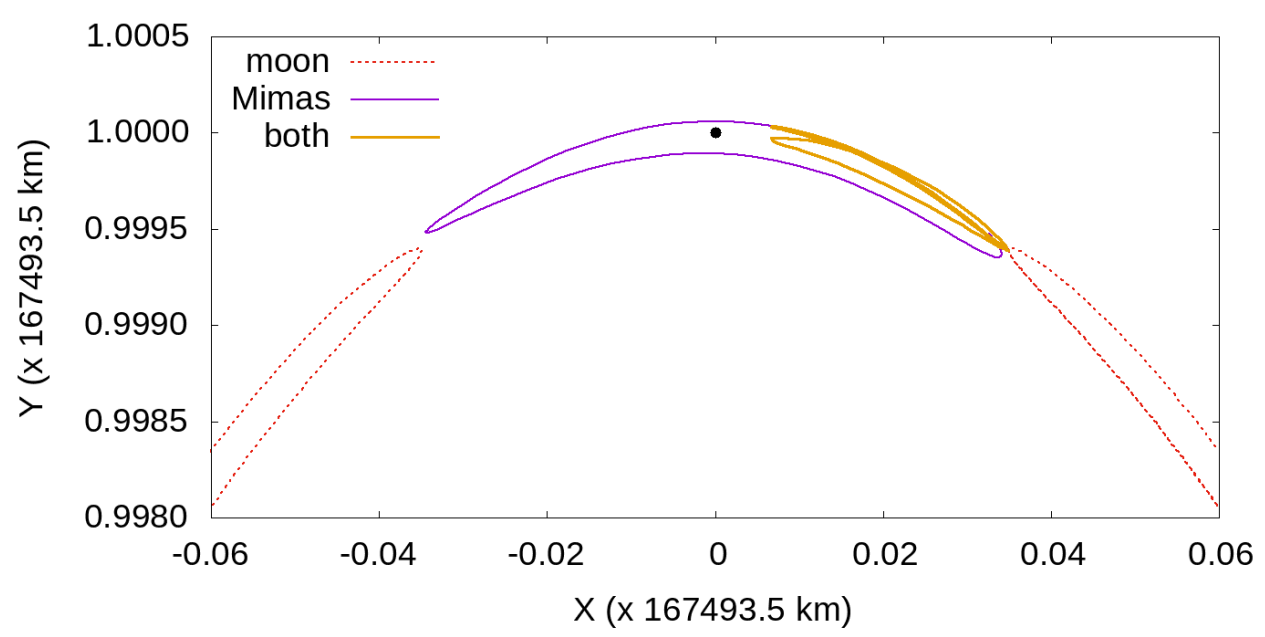

(b)

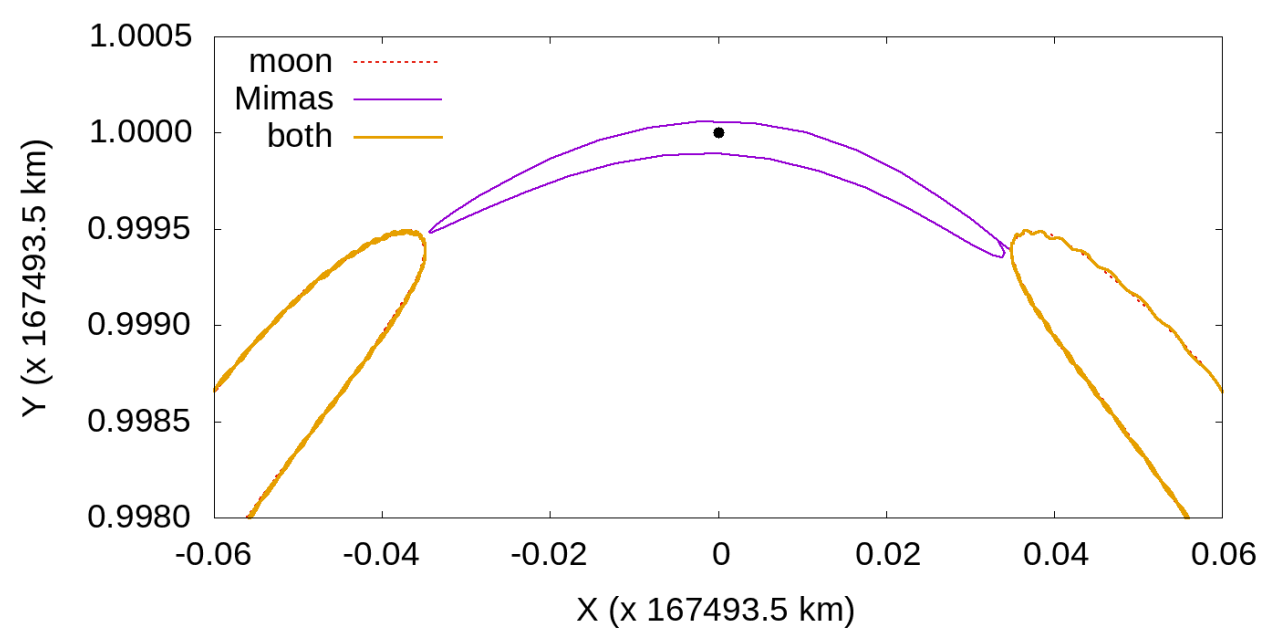

(c)

Fig. 8. Motion of particles initially placed $2^{\circ}$ from the centre of the 7:6 CER site for a) $\mu=10^{-8}$, b) $\mu=10^{-5}$ and c) $\mu=10^{-2}$. When the system is formed by Saturn and Mimas the motion of the particle is represented by a full line (arc), when the system is formed by Saturn and the moonlet its motion is shown in dashed line (horseshoe orbit) and when both satellites are present (Mimas and the moonlet) the motion of the particle can be seen in a thicker full line. 
Table 4. Radius of the moonlets immersed in the centre of each site and its label.

\begin{tabular}{rccc}
\hline \hline & moonlet & radius $(\mathrm{km})$ & label \\
\hline $50 \%$ & \multirow{3}{*}{ radius of Aegaeon } & 0.13 & $0.5 \mathrm{x}$ \\
$100 \%$ & 0.25 & $1.0 \mathrm{x}$ \\
$150 \%$ & & 0.38 & $1.5 \mathrm{x}$ \\
\hline $100 \%$ & \multirow{2}{*}{ radius of Anthe } & 0.50 & $1.0 \mathrm{y}$ \\
$150 \%$ & & 0.75 & $1.5 \mathrm{y}$ \\
\hline $100 \%$ & & 1.40 & $1.0 \mathrm{z}$ \\
$150 \%$ & \multirow{2}{*}{ radius of Methone } & 2.18 & $1.5 \mathrm{z}$ \\
$200 \%$ & 2.90 & $2.0 \mathrm{z}$ \\
$250 \%$ & & 3.63 & $2.5 \mathrm{z}$ \\
\hline
\end{tabular}

The value of the mass production rate enable us to estimate the time $t_{p}$ necessary to the moonlet populates the CER site with a sample of $\mu \mathrm{m}$-sized dust particles (1-10 $\mu \mathrm{m})$ :

$$
t_{p}=\frac{m_{s}}{M^{+}}
$$

where $m_{s}$ is the mass of the site given by [26]:

$$
m_{s}=A_{s}\left(\frac{4}{3} \pi \rho\right) \int_{1 \mu \mathrm{m}}^{10 \mu \mathrm{m}} \pi r^{3} d N
$$

$\rho$ is the density of the particle $\left(\rho=1 \mathrm{~g} / \mathrm{cm}^{3}\right)$ and $A_{s}$ is the surface area of the site.

We assumed the distribution of dust particles to be $d N=C r^{-3.5} d r$ [30, where $C$ is a constant determined by the optical depth [26]

$$
\tau=\int_{1 \mu \mathrm{m}}^{10 \mu \mathrm{m}} \pi r^{2} d N
$$

The optical depth of the $\mathrm{G}$ ring arc was assumed to be $\tau=10^{-5}$, and for the arcs of Methone and Anthe the adopted value is $\tau=10^{-7}$ [4,5].

To determine $A_{s}$ we assumed the site as a ring segment with angular width of $360^{\circ} / m$ and internal and external edges as $\left(a_{c}-W_{c} / 2\right)$ and $\left(a_{c}+W_{c} / 2\right)$ from the centre of the planet, respectively. $A_{s}$ can be written as

$$
A_{s}=\frac{2 \pi}{m} a_{c} W_{c}
$$

The algorithm described above was used to calculate how long the moonlet takes to populate the site.

We also computed the lifetime of the CER site particles through a sample of numerical simulations for a time span of 1000 years. Each site (7:6, 14:15 and 10:11 CER) was composed by a set of particles and an immersed moonlet located in its centre, under the perturbations of Mimas and the gravity coefficients of Saturn. The size of the moonlet was assumed to be proportional to the nominal satellite located in each arc of the Saturn system. Table 4 shows the values of the radius of the moonlets. Moonlets larger than the $2.5 \mathrm{z}$ case have ejection velocities greater than the average velocity of the dust produced by IDPs processes and the equation 15 cannot be applied. We performed numerical simulations without and with both dissipative forces, solar radiation force and plasma drag. The arcs are formed by particles with $1 \mu \mathrm{m}$ and $10 \mu \mathrm{m}$ in radius.

Figure 9 shows the time (in years) versus different sizes of a moonlet immersed in the arc. The curve which shows the time that each moonlet needs to populate the 
arc with a sample of 1-10 $\mu \mathrm{m}$ dust particles (derived from equation 15) was labelled as "production". The time for $90 \%$ of all the particles leave the site or collide with the moonlet without any dissipative force was labeled as "cms". In this case, the arc particles are only under the gravitational perturbation of Mimas and the moonlet and can represent the motion of cm-sized particles if they are in the arcs. For this size range the effects of the dissipative forces can be neglected.

When dissipative forces, solar radiation force and plasma drag, are present in the system, there are two curves labelled as: " $1 \mu \mathrm{m}$ " and "10 $\mu \mathrm{m}$ ". The first one represents the arc populated by $1 \mu \mathrm{m}$ sized particles while the curve labelled " $10 \mu \mathrm{m}$ " refers to the arc populated by $10 \mu \mathrm{m}$ sized particles. Note that these two curves have values for $0.0 \times$ (in the $x$ axis), which means that the arc has none immersed moonlet and its lifetime is derived from Table 3 .

Figure 9a represents the 7:6 CER simulated arc. The "production" curve decreases as the size of the moonlet increases, larger moonlets need less time to populate the arc, as expected. The lifetime of the arc particles, when no dissipative force is present in the system, can be seen in the "cms" curve which shows the first and second regimes, as discussed in section 4 . The first regime corresponds to moonlets which sizes from $0.5 x$ (radius $=0.13 \mathrm{~km}$ ) to $1.5 y$ (radius $=0.75 \mathrm{~km}$ ), the "cms" curve increases up to the size 1.5y. In this regime the particles go further from the moonlet. For larger moonlets $(>1.5 y)$ the particles are confined in the second regime and closer to the moonlet, leading the particles to collisions with them. Both " $1 \mu \mathrm{m}$ " and "10 $\mu \mathrm{m}$ " curves represent the lifetime of the particles when the solar radiation force is acting in the system. The " $10 \mu \mathrm{m}$ " curve is very similar to the "cms" curve, since the solar radiation perturbation causes only small increase in the eccentricity of the $10 \mu \mathrm{m}$ sized particles. The only difference is that the size of the moonlet separating the first to the second regime is $1.0 z$ (radius $=1.40 \mathrm{~km}$ ), larger than for the "cms" curve. Accordingly to Figure 9 a the time for the moonlets produce arc particles is always larger than the lifetime of the particles. Therefore the 7:6 CER arc is a transient arc.

Figure 9p represents the 14:15 CER arc. Both curves, the "production" and the "cms" curves, present almost the same behavior as discussed in Figure $9 \mathrm{a}$. There is one difference, for larger moonlets $(>1.0 z)$ the time to produce dust particles to supply those lost by the arc is smaller than the lifetime of the arc. This arc could survive if larger particles populate it. However, another process would be need to replenish the arc since the IDPs process is able to provide only micrometer dust particles to the arc. The " $10 \mu \mathrm{m}$ " curve presents similar behavior as analysed in Figure 9a, however in this case (Figure 9b) both dissipative forces are present. The "1 $\mu \mathrm{m}$ " curve seems to be independent of the size of the moonlet, the lifetime of the arc populated by $1 \mu \mathrm{m}$ sized particles is about 10 years regardless the size of the immersed moonlet.

Figure 9. represents the 10:11 CER arc. The analysis of the behavior of the curves in Figure $9 \mathrm{p}$ is the same for Figure 98. The arcs of Anthe and Methone have similar dynamical behavior.

\section{Discussion}

The arcs in Saturn system have immersed satellites on them. The G ring arc has the small satellite Aegaeon, while the arcs of Anthe and Methone have both satellites Anthe and Methone present. These arc particles are also under the resonant effects of the satellite Mimas. Aegaeon, Anthe and Methone are trapped in a 7:6, 10:11 and 14:15 CER with Mimas. The population of these arcs is mostly formed by $\mu$ m-sized particles, which are disturbed by dissipative forces such as the solar radiation force. 


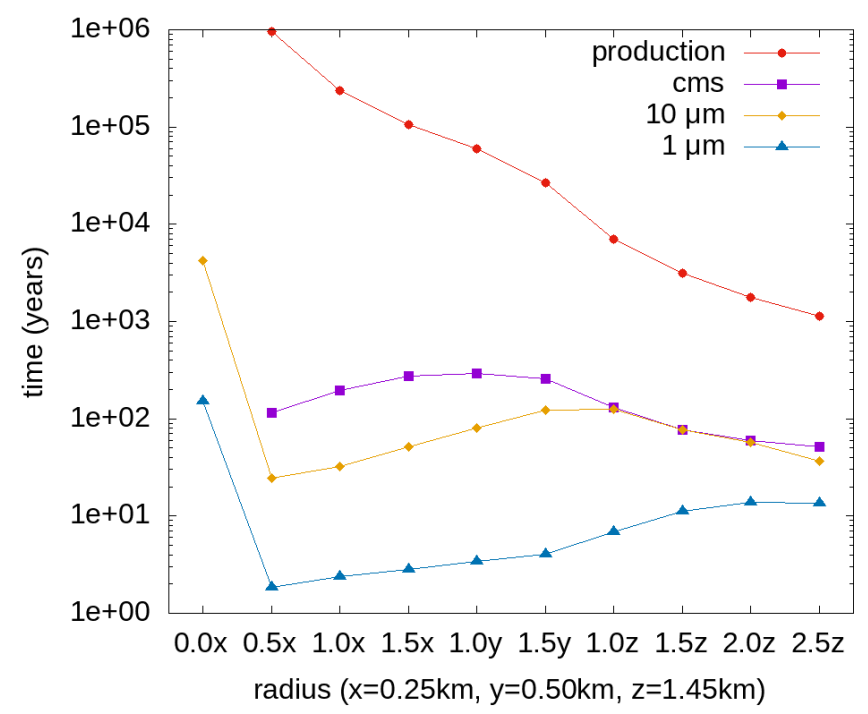

(a)

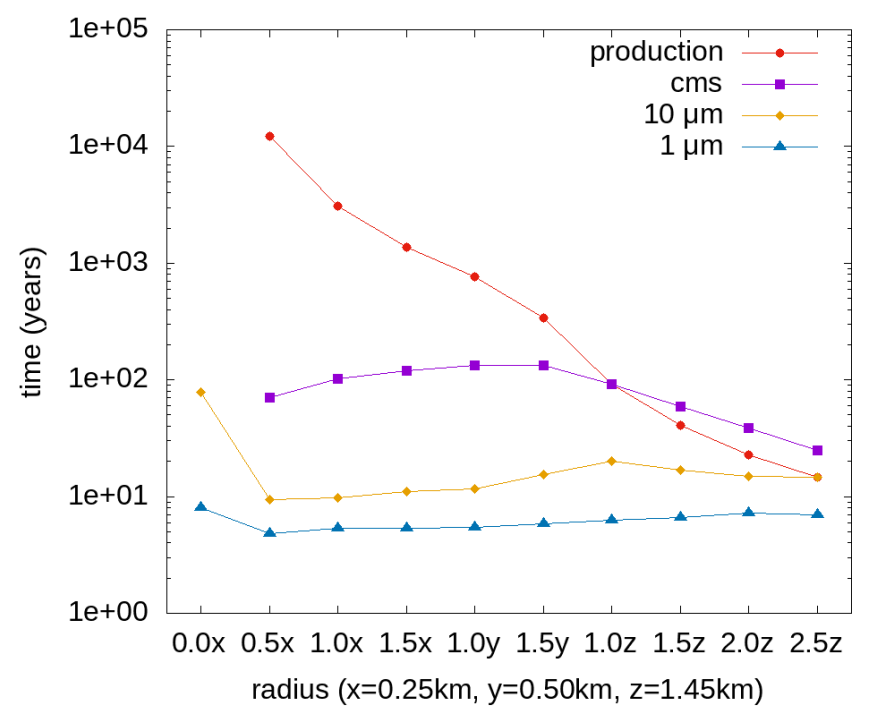

(b)

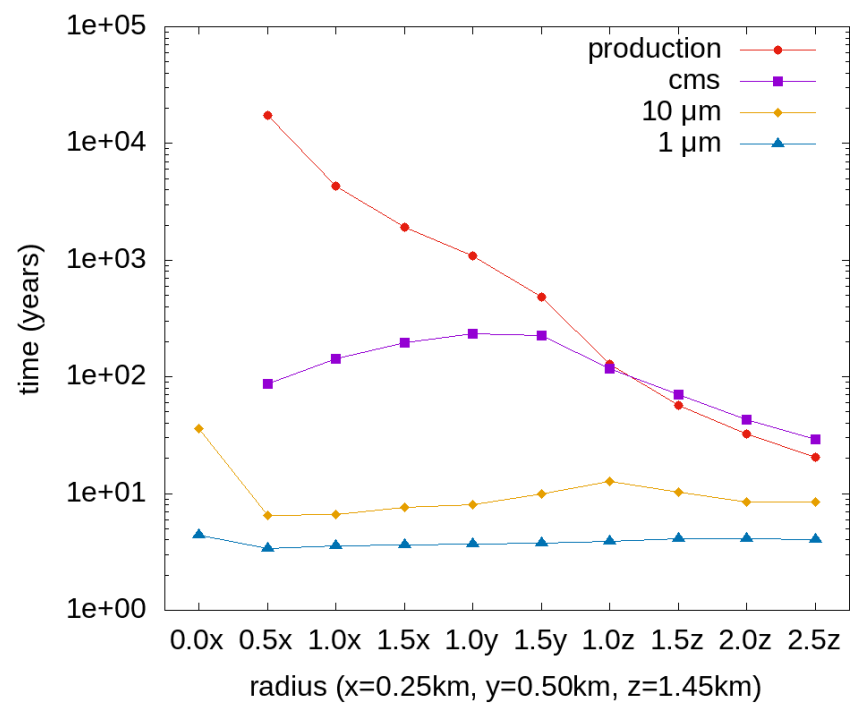

(c)

Fig. 9. Time (in years) versus size of the moonlet. This figures shows the time for the moonlet produces material ("production") and removes it for two cases, without ("cms") and with dissipative forces. For the last case two samples of particles were analysed: $1 \mu \mathrm{m}$ ("1 $\mu \mathrm{m}$ ") and $10 \mu \mathrm{m}$ ("10 $\mu \mathrm{m} ")$ sized particles. Each plot represents each site: a) 7:6 CER, b) 14:15 CER and c) 10:11 CER. 
In this work we analysed the lifetime of these arcs under the gravitational effects of Saturn, Mimas, the small satellites and dissipative forces. For the arcs of Methone and Anthe, since they are closer to Enceladus, the effects of the plasma drag have to be taken into account. As a result we found that the $1 \mu \mathrm{m}$ sized particles live longer in the $\mathrm{G}$ ring arc (about 150 years) while in the arcs of Anthe and Methone they can last at most one decade. Larger particles $(10 \mu \mathrm{m}$ in radius) located in the $\mathrm{G}$ ring arc, Methone and Anthe arcs have lifetimes larger than 1000 years, about 80 and 35 years, respectively.

An immersed moonlet can affect the arc population in two ways. Firstly, the moonlet can supply the arc population with particles released from its surface after a collision with interplanetary objects. The moonlet can be the source of the arc. By the other hand, the moonlet can disturb the particles and provokes collisions and ejections from the arc. The moonlet can also be the sink of the arc. Therefore we analysed a hypothetical scenario by assuming a moonlet of different sizes present in each arc. We calculated the mass production of each moonlet and the orbital evolution of the particles, and consequently their lifetimes, under the effects of the moonlet.

Our results, after a sample of numerical simulations, have shown that a set of particles under the gravitational effects of Mimas and a hypothetical moonlet can perform three different motions. When the moonlet has mass ratio between $10^{-10}$ to $10^{-7}$ the particle stays in an arc larger than if only Mimas was present in the system. As the moonlet mass increases up to $10^{-3}$, the moonlet reduces the motion of the particle. It stays in half part of the arc. For moonlets larger than $10^{-3}$ the particle performs a horseshoe orbit disturbed by Mimas.

By comparing the lifetime of the arcs (without and with the dissipative forces) and the mass production due to the moonlets we found that all three arcs are transient. The dissipative forces dominate the micrometric particles dynamics, regardless of their size. They survive in the arc up to a few hundred of years while the time for a moonlet replenish the arc is always longer than the particles lifetime, according to our dynamical model. A cm-sized population could survive in the arc for a longer period of time however these particles cannot be supplied by the IDPs process. In general, we found that the arcs are rapidly depleted of micrometric dust particles and they tend to be transient structures.

\section{Acknowledgements}

The authors thank Fapesp (2016/24488-0, 2016/24561-0 and 2018/23568-6) and CNPq (309714/2016-8) for the financial support. This study was financed in part by the Coordenação de Aperfeiçoamento de Pessoal de Nível Superior - Brasil (CAPES) Finance Code 001.

GM and SMGW divided the work of writing and the numerical simulations.

\section{References}

1. C.C. Porco, Science 253, (1991) 995

2. I. de Pater, S.G. Gibbard, E. Chiang, H.B. Hammel, B. Macintosh, F. Marchis, S.C. Martin, C. Shuleen H.G. Roe, M. Showalter, Icarus 174, (2005) 263

3. M.M. Hedman, J.A. Burns, M.S. Tiscareno, C.C. Porco, G.H. Jones, E. Roussos, N. Krupp, C. Paranicas, S. Chris, Science 5838, (2007) 653

4. M.M Hedman, C.D. Murray, N.J. Cooper, M.S. Tiscareno, K. Beurle, M.W. Evans, J.A. Burns, JA, Icarus 199, (2009) 378

5. M.M. Hedman, N.J. Cooper, C.D. Murray, K. Beurle, M.W. Evans, M.S. Tiscareno, J.A. Burns, B. Sicardy, Icarus 207, (2010) 433 
6. N.J. Cooper, C.D. Murray, M.W. Evans, MW K. Beurle, R.A. Jacobson, C.C. Porco, Icarus 195, (2008) 765

7. J.N. Spitale, R.A. Jacobson, C.C. Porco, W.M. Owen Jr, The Astronomical Journal 132, (2006) 692

8. P.C. Thomas, J.A. Burns, M.M. Hedman, P. Helfenstein, S. Morrison, M.S. Tiscareno, J. Veverka, Icarus 226, (2013) 999

9. M.E. Moutamid, B. Sicardy, S. Renner, Celestial Mechanics and Dynamical Astronomy 118, (2014) 235

10. G. Madeira, R. Sfair, D.C. Mourão, S.M. Giuliatti Winter, Monthly Notices of the Royal Astronomical Society 475, (2018) 5474

11. K.L. Sun, M. Seiß, M.M. Hedman, F. Spahn, Icarus 284, (2017) 206

12. S. Renner, B. Sicardy, D. Souami, B. Carry, C. Dumas, Astronomy \& Astrophysics 563, (2014) A133

13. S.M. Giuliatti Winter, G. Madeira, R. Sfair, Monthly Notices of the Royal Astronomical Society (2019), To be submitted

14. B. Sicardy, Icarus 89, (1991) 197

15. W.H. Press, S.A. Teukolsky, W.T. Vetterling, B.P. Flannery, Numerical recipes in Fortran 77: the art of scientific computing (Cambridge university press Cambridge, 1992)

16. C.D. Murray, S.F. Dermott, Solar system dynamics (Cambridge university press, 1999)

17. K.M. Ellis, C.D. Murray, Icarus 147, (2000) 129

18. J.E. Chambers, Monthly Notices of the Royal Astronomical Society 304, (1999) 793

19. S. Renner, B. Sicardy, Celestial Mechanics and Dynamical Astronomy 94, (2006) 237

20. F. Mignard, Planetary Rings (IAU, 1984) 333-366

21. D.P. Hamilton, A.V. Krivov, Icarus 123, (1996) 503

22. R. Sfair, S.M. Giuliatti Winter, Astronomy \& Astrophysics 505, (2009) 845

23. B.H. Mauk, D.C. Hamilton, T.W. Hill et al., Saturn from Cassini-Huygens (Springer, 2009) 281-331

24. M. Banaszkiewicz, H.J. Marek, K. Scherer, Icarus 107, (1994) 358

25. A.R. Poppe, Icarus 264, (2016) 369

26. R. Sfair, S.M. Giuliatti Winter, Astronomy \& Astrophysics 543, (2012) A17

27. F. Spahn, N. Albers, M. Hörning, S. Kempf, A.V. Krivov, M. Makuch, J. Schmidt et al., Planetary and Space Science 54, (2006) 1024

28. A.V. Krivov, M. Sremčević, F. Spahn, V.V. Dikarev, K.V. Kholshevnikov, Planetary and Space Science 51, (2003) 251

29. D. Koschny, E. Grün, Icarus 154, (2001) 391

30. J.E. Colwell, L.W. Esposito, Journal of Geophysical Research: Planets 98, (1993) 7387 